\title{
Dégâts des bruches sur le pouvoir germinatif des graines de quatre variétés de Niébé infesté pendant 60 jours à Ngandajika
}

\author{
Robert Mukendi K , Richard Ntanga N¹, Stephane Kaseba K, Tshiamala N¹, Alphonse Kamukenji1, \\ Germain Mpoyi K² \\ ${ }^{1}$ Institut National d'Études pour la Recherche Agronomiques (INERA). Centre de Recherche de Ngandajika \\ République Démocratique du Congo \\ ${ }^{2}$ Institut Supérieur d'Études Agronomiques de Mukongo (I.S.E.A. Mukongo) Mbuji Mayi, République Démocratique \\ du Congo \\ Auteur email correspondante : mukendikam@yahoo..fr
}

Original submitted in on $13^{\text {th }}$ October 2015. Published online at www.m.elewa.org on $29^{\text {th }}$ February 2016 http://dx.doi.org/10.4314/jab.v98i1.8

\section{RÉSUMÉ}

Objectif : L'objectif de cette étude est de déterminer l'impact des dégâts des bruches sur le pouvoir germinatif des graines de niébé et d'identifier des variétés résistantes ou tolérantes aux attaques de bruches pendant le stockage. Le bruche (Callosobruchus maculatus) est un petit coléoptère doté de taches sombres sur les élytres. C'est un parasite qui s'attaque aux graines pendant l'emmagasinage.

Méthodologie et résultats : Au cours de ce travail, nous avons étudié le comportement de quatre (4) variétés améliorées de niébé (Yamashi, Mujilanga, IT82D-889 et Diamant) sélectionnées par l'Institut National d'Études pour la Recherche Agronomiques centre de Ngandajika sur le développement de Callosobruchus maculatus infesté dans les graines de ces variétés. Cette étude était menée in vitro au laboratoire du centre de Recherche de I'INERA Ngandajika. Les résultats révèlent que le taux d'accroissement des bruches infestés pendant 60 jours varie de $50.41 \%$ à $91.99 \%$. Et la variété Diamant à présenté un pouvoir germinatif beaucoup plus faible $0.75 \%$ que les trois autres variétés (Mujilanga $7.75 \%$, Yamashi $8.25 \%$ et IT82D-889 12.75\%.

Conclusion et application des résultats : Cette étude a permis de conclure que la variété Diamant a perdu le pouvoir germinatif après une durée de conservation de 60 jours.

Ces résultats suggéreraient que pour une période de conservation après récolte, les graines de niébé de la variété Diamant ne peuvent plus être considérées comme semences. II est par conséquent important de demander aux producteurs de semences de cette variété de ne pas utiliser la semence de cette variété après 60 jours de conservation sans traitement phytosanitaire et d'utiliser ainsi la semence fraichement récoltée. Mots clés : pouvoir germinatif, résistance variétale.

\section{ABSTRACT}

Objective: The objective of this study is to determine the impact of damage from weevils on the germination of the seeds of cowpea and identify varieties resistant or tolerant to attack by weevils during storage. Callosobruchus maculatus. The weevil (Callosobruchus maculatus) is a small beetle with dark spots on the wing covers. This parasite attacks the seeds during storage. 
Methodology and results: During this work, we studied the behaviour of four (4) improved varieties of cowpea (Yamashi, Mujilanga, IT82D-889 and Diamond) selected by the National Research Institute for Agronomic Research centre on the development Ngandajika of Callosobruchus maculatus infested in the seeds of these varieties. This study was conducted in vitro laboratory research centre INERA Ngandajika. The results reveal that the rate of increase of weevils infested for 60 days ranged from $50.41 \%$ to $91.99 \%$. Diamond and variety germination presented a much lower $0.75 \%$ than the other three varieties (Mujilanga $7.75 \%, 8.25 \%$ and IT82D Yamashi-889 12.75\%.

Conclusion and application of results: This survey permitted to conclude that the variety Diamant lost the germinating power after a length of conservation of 60 days. These results would suggest that for one period of conservation after harvest, the seeds of cowpea of the variety Diamant could not be considered more like seeds. It is therefore important to ask the producers of seeds of this variety to not to use the seed of this variety after 60 days of conservation without phytosanitary treatment and to use the freshly harvested seed.

Key words: germinating power, variety resistance.

\section{INTRODUCTION}

Le niébé Vigna -Unguiculata $(\mathrm{L})$ Walp, est la légumineuse à graine la plus importante avec une production mondiale estimée à 3 millions de tonnes sur une superficie de 12,5 millions d'hectares (SINGH et al., 1997). Le niébé est consommé par près de 200 millions de personnes en Afrique tropicale (NTANG, 2004). II constitue l'une des cultures vivrières de base en Afrique Occidentale et centrale (MULEBA et al., 1997). En RD Congo il est consommée dans les deux Kasaï, ainsi que dans une grande partie de la province du Bas-Congo et Bandundu), sa capacité de fixer symbiotiquement l'azote atmosphérique par ses racines a partir de bactéries du genre Rhizobium lui permet d'être à la fois riche du point de vue nutrition humaine, et moins cher pour l'amélioration de la fertilité du sol (BOER, 1989; BADO, 1999 et 2002). C'est une culture relativement résistante à la sécheresse. La symbiose avec les bactéries indigènes(Ryzobia) lui permet non seulement de s'adapter aux sols pauvres, mais également d'améliorer la composition chimique facilement assurée par les engrais chimiques qui sont rares et onéreux pour les petits paysans (Wey et Obaton, 1978). Sa teneur en protéines 23\% fait que le niébé soit indiqué pour améliorer l'alimentation des populations des zones rurales de la RD Congo en général et de la province du Kasaï oriental en particulier où les protéines animales sont rares, alors que le manioc et le mais constituent la base de l'alimentation (Dakara, 1985, munyuli et al., 2007). Les insectes constituent la principale contrainte de ce faible rendement. Les aphides, les pucerons, les thrips et les punaises endommagent le niébé au champ tandis que les bruches endommagent la récolte stockée. Dans le pays en développement les pertes post- récoltes sont considérables et les conditions climatiques tropicales et Intertropicales favorisent les dégâts et altérations des produits stockés. Les bruches (Callosobruchus maculatus) se nourrissent des graines de niébé en y perforant des trous ronds à l'intérieur des graines et consomment les réserves nutritives contenues dans les cotylédons causant ainsi des pertes quantitatives et qualitatives (Alzouma, 1994),Huignard et al., 1995, Onukola C., 2003). La destruction de $50 \%$ des graines de niébé au Brésil entraine une perte de $50 \%$ de la valeur marchande (Bastos, 1977 ; cité par Alzouma, 1991). Au Nigeria, les pertes avoisinent 2900 T/an (Caswell, 1973). Dans ce même pays qui est le plus grand producteur mondial (900.000 T/an). Singh et al, (1983) évaluent les pertes à plus de 30 millions de dollars. Au Niger, CL, maculatus entraine la perte de $30 \%$ de la production nationale (Alzouma, 1995). Au Sénégal, 90\% des graines peuvent être endommagées après 6 mois de stockage (Seck. 1992). L'infestation des graines affecte leur valeur nutritive et leur qualité organoleptique et peut finalement rendre le stock inconsommable. Devant le manque de devises de nombreux pays en développement en général, de la RD Congo et de la province du Kasaï-Oriental en particulier l'importation des pesticides et considérant la pollution qu'ils entrainent ainsi que le faible niveau des connaissances techniques de la population, il 
importe d'étudier le comportement de certaines variétés vis-à-vis de l'attaque de bruche et de leur résistance (Regnault-Roger, 2002; Ketoh et al.,2005).Le bruche est le principal ennemi des produits emmagasinés; son aire des distributions s'étend à toutes les régions du globe. Lors d'infestation graves, le pourcentage de graines endommagées peut atteindre $30 \%$ après 6 mois de conservation. L'adulte est un coléoptère court et trapu dont les élytres portent des marques plus sombres. Après éclosion, la larve pénètre à l'intérieur de la graine, où elle poursuit son développement.

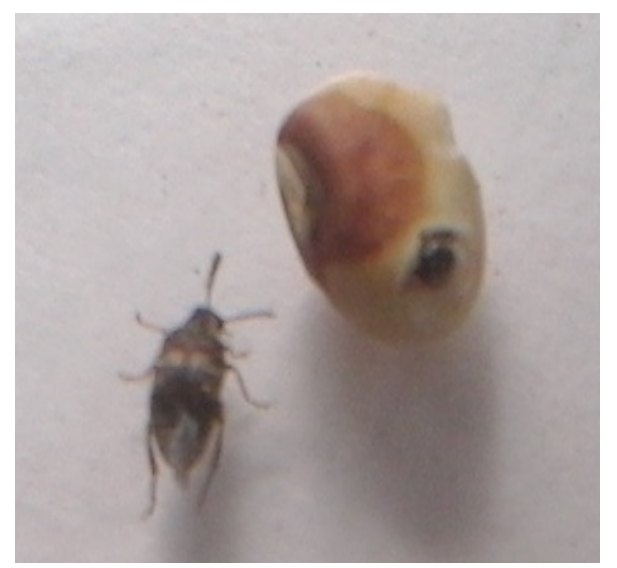

Image 1 : Bruches+niébé endommagé

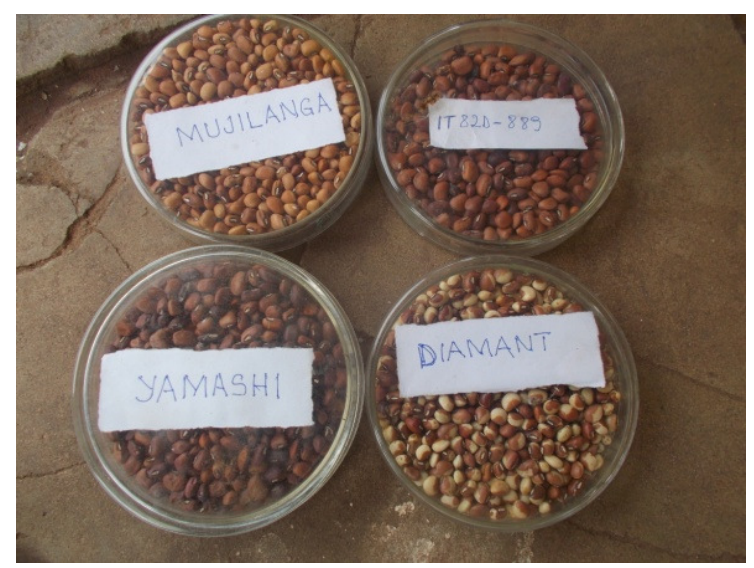

Image 2 : Les quatre variétés comparées.
La présence de ces trous constitue un critère d'identification des graines infestées. Le stade adulte a une durée de vie de 5 à 8 jours, tandis que le cycle de vie totale de l'insecte dure environ 30 jours (Ouedraogo, 1978). L'infestation des gousses débute aux champs, mais la population des bruches croit rapidement suite au décorticage après un dépôt direct des œufs sur les graines. Après éclosion des œufs, les larves pénètrent dans les graines ou elles complètent leur développement. Les adultes vivent 5 à 10 jours durant lesquels la femelle est fécondée. Celle-ci dépose environ une Cinquantaine d'œufs sur les graines. Le cycle biologique de l'insecte est de 3 à 4 semaines. En conséquence, à partir d'une modeste infestation initiale, la plupart des graines

\section{MATÉRIEL ET MÉTHODES}

Les graines des variétés de niébé: Quatre lots de graines des variétés Yamashi, Mujilanga, IT82D-889 et Diamant issus de la campagne culturale 2013 ont été choisis lors de cette étude. Les graines issues des variétés pures de niébé ont été fournies par le Programme National Légumineuses de l'Institut National d'Études et Recherche Agronomique (INERA/Ngandajika). II s'agit des variétés suivantes: Diamant, Yamashi, Mujilanga et IT82D-889.Ils ont été peuvent se retrouver après deux mois de stockage, correspondant à deux cycles de reproduction, avec des trous ou des œufs sur elles. Les trous occasionnés par le bruche favorisent le développement des moisissures et autres parasites. L'effet additif des différents dégâts peut occasionner des pertes économiques de $100 \%$, car avec des pertes pondérales de 20 à $30 \%$, les pertes économiques sont de $100 \%$ et les graines deviennent impropres à la consommation (Ndiaye, 1991). La présente étude se propose de déterminer parmi les quatre variétés, celle qui sera moins attaquée après une durée de 60 jours d'infestation artificielle.

utilisés pour comparer l'influence du temps ou la durée de stockage dans les conditions d'infestation forcée (artificielle) sur le pouvoir germinatif de niébé. Il s'agit des variétés testées au laboratoire dans des boites de pétri en verre afin d'éviter l'attaque des insectes ou d'autres rongeurs. La durée de l'expérience était de 60 jours qui vont depuis l'installation jusqu'au dépouillement. 


\section{Mukendi et al. J. Appl. Biosci. 2016 Dégâts des bruches sur le pouvoir germinatif des graines de quatre}

variétés de Niébé infesté pendant 60 jours à Ngandajika

Accouplement des bruches (Callosobruchus maculatus) et infestation des traitements : Ici il s'agit tout simplement de déterminer le nombre des bruches et leurs espèces à charger dans chaque boite de pétri selon les blocs et les traitements. Un sac de semence de niébé très attaqué à servi de cadre de prélèvement des bruches d'espèce Callosobrus maculatus généralement comme chez tous les insectes les males et femelles ont été identifiés sur base de leur taille. C'est-à-dire la femelle est grande, tandis que le male est petit, le nombre de 4 bruches représentant deux couples à été choisi pour l'infestation de 100 graines saines.

Le comptage des bruches et le test de germination : Le comptage a été réalisé selon la méthode suivante:

\section{RÉSULTATS ET DISCUSSION}

Caractéristiques morphologiques et technologiques : Les caractéristiques morphologiques et technologiques des graines des différentes variétés sont présentées dans le tableau 1. Pour la détermination de la couleur des graines, le catalogue des variétés a été utilisé. II donne la dépouillement du contenu de la boite de pétri afin de procéder au comptage de chaque individu de bruche rencontré puis, calculer le taux de natalité et l'accroissement de la population, en se référant au nombre de couple infestés sur le total multiplié par 100. Les analyses des variances sont effectuées avec le logiciel STATISTIX au seuil de $5 \%$.

Le test de germination : Ce test à été effectué après avoir semé toutes les 100 graines contenues dans la boite de pétri. A la germination, faire le rapport entre le nombre de graines germées sur le total multiplié par 100

couleur des différentes variétés cultivées en République Démocratique du Congo. Celle des variétés étudiées varie de beige pour la variété mujilanga, a rouge foncé pour la variété IT 82D-888 en passant par la couleur tachetée pour la variété Diamant.

Tableau1 : Caractéristiques morphologiques et cycle biologique des graines de niébé

\begin{tabular}{l|c|c|c}
\hline Variétés & Couleur & $\begin{array}{c}\text { Cycle végétatif } \\
\text { (Jours) }\end{array}$ & $\begin{array}{c}\text { Poids de 100 } \\
\text { graines(g) }\end{array}$ \\
\hline DIAMANT & tachetée Bicolore & $70-75$ & $110-120$ \\
YAMASHI & Mauve & $70-80$ & $100-110$ \\
MUJILANGA & Beige & $75-80$ & $100-110$ \\
IT82D-889 & Rouge foncé & $65-70$ & $100-110$ \\
\hline
\end{tabular}

Influence de la variété et du temps de stockage : Les temps de conservation des différentes variétés de niébé sont représentés dans le tableau 2. La durée de conservation correspond au temps entre le début de l'infestation des graines par les bruches et celui ou elles sont prêtes à être dépouillé à 60 jours pour les variétés identifiées. La variété Diamant présente une attaque la plus sévère soit $99.25 \%$ tandis que la variété IT82 D889 présente un taux d'attaque de $87.25 \%$. Ces résultats montrent tout d'abord que la variété a une influence sur l'attaque d'infestation des bruches sur les graines de niébé. Elle est corrélée à la durée de conservation des graines, qui dépend de fois à la structure chimique de chaque génotype.

Tableau 2: Taux de natalité, de mortalités et les accroissements des bruches sur ces quatre variétés.

\begin{tabular}{l|c|c|c|c}
\hline Traitements & Taux de Natalité & Taux de mortalité & Accroissement en \% & Accroissement par jour \% \\
\hline Diamant & 98.37 & 6.38 & 91.99 & 1.53 \\
Mujilanga & 77.28 & 8.41 & 68.87 & 1.14 \\
Yamashi & 54.56 & 4.15 & 50.41 & 0.89 \\
IT 82 D-889 & 84.88 & 6.08 & 78.8 & 1.31 \\
\hline
\end{tabular}

Les résultats obtenus révèlent que le taux d'accroissement des bruches infestés pendant 60 jours varie de $50.41 \%$, à $91.99 \%$, tandis que le taux par jour varierait de $0.89 \%$ à 1.53 . Ce qui situe les quatre variétés dans la plage des graines infestées et la vitesse de reproduction de bruches dans chaque variété par jour. La variété Diamant semble être la plus attaquée donc ayant beaucoup des trous, tandis que IT82D-889 est la variété la moins attaquée des quatre. Ce facteur de résistance est peut être essentiellement génétique, mais 


\section{Mukendi et al. J. Appl. Biosci. 2016 Dégâts des bruches sur le pouvoir germinatif des graines de quatre variétés de Niébé infesté pendant 60 jours à Ngandajika}

peut aussi dépendre des conditions agro climatiques. Quand au poids de 100 graines, la variété Diamant possède les graines lourdes par rapport aux autres variétés. Ces résultats montrent tout d'abord que la variété a une influence sur l'attaque d'infestation des bruches sur les graines de niébé.

\section{Graphique $\mathbf{N}^{\circ} 1$}

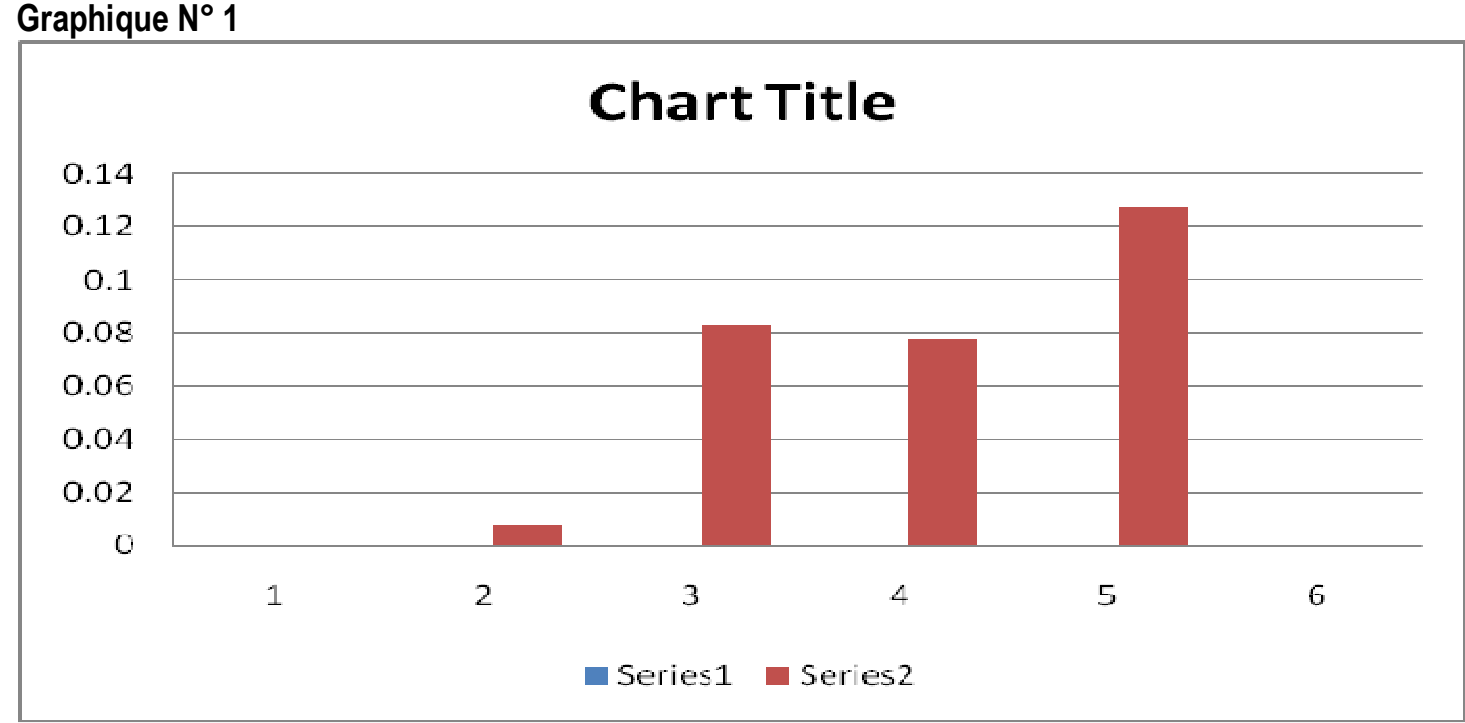

\section{L'influence des variétés sur le taux de germination}

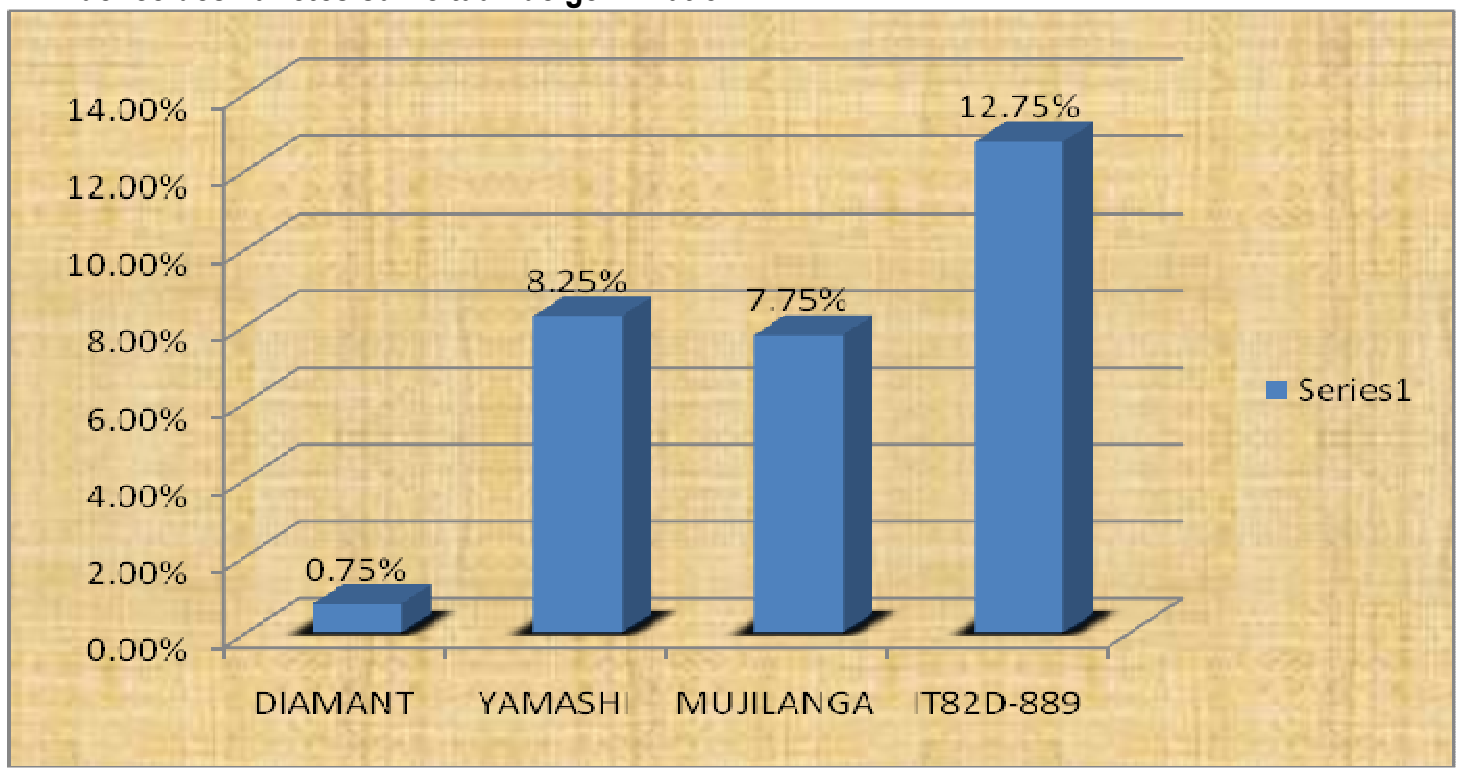

\section{DISCUSSION}

Les résultats obtenus sur les différents paramètres étudiés font ressortir une certaine variabilité entre les variétés testées. II ressort de l'analyse de nos résultats que le taux d'accroissement des bruches pendant 60 jours varie de $50.41 \%$ à $91.99 \%$, tandis que le taux par jour varierait de $0.89 \%$ à $1.53 \%$ ce qui prouve que la reproduction des bruches est très rapide. Concernant les traitements, les Variétés Mujilanga, Yamashi et IT 82D889 semblent résister aux attaques, ensuite nous remarquons que la variété Diamant est mieux attaquée et a perdu considérablement le pouvoir germinatif. Cette situation a été démontré par certains auteurs et elle serrait du peut être aux effets génétiques. 


\section{CONCLUSION}

Cette étude sur les dégâts des bruches sur le pouvoir de germination des graines de quatre variétés de niébé infestées pendant 60 jours de stockage a montre que la variété Diamant est la variété la plus attaquée avec un taux de mortalité de plus de 6,38\%, tandis que les autres variétés (mujilanga, yamashi et IT82D-886) se sont assez bien comportées avec un taux de mortalité allant de 4,15 a $8,41 \%$. Cette expérience permet d'induire que la

\section{REMERCIEMENT}

Nous remercions le centre de Recherche de l'INERA (Institut National d'Étude pour la Recherche Agronomique) centre de Ngandajika, son Directeur Ir.

\section{REFERENCES BIBLIOGRAPHIE}

Alzoumai, et Huignardj. (1981). Données préliminaires sur la biologie et le comportement de ponte dans la nature de Bruchidus atralineatus pic (coléoptères ponte dans la nature de bruchidus atralineatus pic (Coléoptères bruchideae) dans une zone sud, Sa, Acta hélienne au Niger Oecol, Appel, $2: 391-400$ spreng. (Poaceae), and the wasp Dinarnus barolis Rondani (hymenoptera Ptetonolidae), stored Prod Ros, 41:363-37.

Alzouma I.1994: Progrès récents enregistrés sur la connaissance et le contrôle des coléoptères Bruchidae ravageurs des légumineuses alimentaires au Sahel séminaire sur la lutte intégrée au Sahel du 03 au 09 Avril : 393. Dakar (Sénégal).

Alzouma I., 1995 : Connaissance et contrôle des colcxy f c'.s Hrnchidre r; l.aseurs de; légumineuses alimentaires au Sahel. Sahel Integrated Pest management (I.P.M)/Gestion Phytosanitaire Intégrée. Revue Institut CILSS dc Sahel. NC 1Févr. 1995, pp. 2-16.

Bado B. V., 1999. Les contributions en azote des légumineuses et des amendements organiques : une évaluation quantitative par le 15N, 67p.

Bado B. V., 2002. Rôle des légumineuses sur la fertilité des sols ferrugineux tropicaux des zones guinéennes et soudanienne du Burkina Faso. Thèse de l'Université Laval, http://www.theses.ulaval.ca. 197 p, téléchargé le 10/11/07.

Boer D. L., 1989. Entomologie appliquée Tome 3 (arachide + niébé), CILSS/CENTRE AGRYMET, $75 p$. sensibilité de la variété Diamant peut être traduite par les effets génétiques. Les résultats de cette étude suggéreraient qu'après une période de 60 jours de conservation après récolte de niébé, les graines de la variété Diamant perdent sensiblement le pouvoir germinatif.

Olivier Mulamba et son coordonnateur de Recherche, Dr. Ir. Amand Mbuya kankolongo pour nous avoir accordé un bon cadre de travail.

Caswell G. H., 1973: The impact of infestation on commodities Stored Prod. Inf 2.5. 19

Huignard J. 1996: Biological control of Bruchid insect pest of Cowpea in West Africa Rapport final contrat CEE (1993 - 1996) 142 p.

Ketoh GK, Koumogbo HK and Glitho I.A., 2005 inhibition of Collosobruchus maculatus (F) (coleopteran bruchidae) development with essential oil extracted from cymbopogons achaemathus $L$.

Muleba NO, Dablre CO, Suh JO N, Drabo T., Ouedraogo JO 'R., 1997; Technologies for cowpea production based on genetic and environmental manipulations in the Semi-Arid Tropics. Technology options for sustainable agriculture in Sub-Saharan Africa, OAU/STRC-SAFGRAD, pp. $195-206$.

Muleba NO, 1988. Association niébé/céréale dans les zones semi-arides. In : État de la recherche sur la culture du niébé en Afrique centrale et occidentale semi-aride, RENACO, IITA/SAFGRAD, Ibadan, Nigeria, pp. 14 - 17.

Munyulu T.M.B., Luther G.C. et Kyamanywa, S.( 2007). Effets of cowpea cropping systems and insecticides on arthrod predators in Uganda and Democratic Republic of the Congo. International journal of crops Protection, 26 114-126.

Niang I, SO, 2004. Cowpea productivity improvement In the African Agricultural Technology Foundation (AATF).

Okunola C, O., (2003), Use of melon seed oil for the control of bruchid damage.

Ouedraogo AP, Sous, Sanon A et Monge JP 1996 Influence of temperature and humidity on population of $\mathrm{C}$. maculates and its parasitoids, 
dinarmus basulus (pteromalidae) in two of Burkina Faso- bull of enton- Res 86,695-702?

Seck D., 1992: Importance économique et développement d'une approche de lutte intégré contre les insectes ravageurs des stocks de maïs, mil et niébé en milieu paysan Proceedings, deuxième séminaire sur la lutte intégré contre les ennemis des cultures vivrières dans le sahel. Bamako(Mali),2-4 Janvier 1990, pp, $31 \% 355$.

Seck D., Gaspar CH., 1992 : Efficacité du stockage du niébé $(\mathrm{J} \$, \mathrm{~V} / \mathrm{L} \sim$ utlguicl//tttcr (L) IYalp) et-; fûts métalliques hermétiques comme méthode alternative de contrble de C'crlk>sohrlicl7rrs nmcnkrrm F. (Coléoptères bruchidae) en Afrique Sahélienne bled. Fac. Landbonww. Univ. Gent. 57/3a., pp. 75 I-757.

Singh B.B., Chambliss O.L.and Sharma B (1997). Recent advances in cowpea breeding In: B.B. Singh, D.R. Mohan Raj, K.E. Dashiell, and I.E.N. Jackai (Eds). Advances in cowpea research co publication of International Institute of Tropical Agriculture (IITA) and Japan International Research Center for Agricultural (JIRCAS), IITA, Ibadan Nigeria, p. 30 -49.

Wey J, Obaton M. 1978. Incidence de quelques techniques culturales sur l'activité fixatrice d'azote et le rendement de l'arachide Agronomie Tropicale, 33(2). 129-135. 